\title{
Higher dimensional non-Kerr black hole and energy extraction
}

\author{
Sushant G. Ghosh*1,2, 团 and Pankaj Sheoran ${ }^{1, \text { 团 }}$ \\ ${ }^{1}$ Centre for Theoretical Physics, \\ Jamia Millia Islamia, New Delhi 110025, India \\ ${ }^{2}$ Astrophysics and Cosmology Research Unit, School of Mathematical Sciences, \\ University of KwaZulu-Natal, Private Bag 54001, Durban 4000, South Africa
}

\begin{abstract}
We investigate the properties of the horizons and ergosphere in a rotating higher dimensional (HD) deformed Kerr-like black hole. We also explicitly bring out the effect of deformation parameter $\epsilon$ and the extra dimension on the efficiency of the Penrose process of energy extraction from a black hole. It is interesting to see that the ergosphere size is sensitive to the deformation parameter $\epsilon$ as well as spacetime dimensions $D$. This gives rise to a much richer structure of the ergosphere in a HD non-Kerr black hole, thereby making the Penrose process more efficient compared with that of the four-dimensional Kerr black hole.

PACS numbers: 04.70.Bw, 04.50.Gh
\end{abstract}

\section{INTRODUCTION}

The rotating black hole are formed when a star can no longer support itself against its own gravitational collapse, thereby compressing to a point. Energy extraction from a rotating black hole interests us not only as engines of relativistic jets from active galactic nuclei and quasars [1] but also as fundamentals of black hole physics [2]. An interesting process called the Penrose process allows one, in principle, to extract energy from a rotating black hole [3] and relies on conservation of momentum and energy. In the Penrose process, one shoots a massive particle inside the ergosphere, and then it splits into two particles, one of which has negative energy and one of which has positive energy. Penrose showed that the negative energy particle would go down the black hole, but the positive energy particle could escape, carrying with it more energy than it came in with. It turns out the negative energy particle will slow down the spinning of the hole and reduce its energy, and thus it provides an important method to extract energy from a black hole [4 8]. Recently the Penrose process was also extended to the five-dimensional (5D) supergravity rotating black hole [9], to higher dimensional black holes and black rings [10], to the Hořava-Lifshitz gravity black hole [1], to the Kerr-NUT black hole 12], and also to a rotating black hole with a global monopole 13 .

Motivated by examining the no-hair theorem, Johannsen and Psaltis 14 recently applied the NewmanJanis [15] complex transformation to the deformed Schwarzschild solution [16] and constructed a Kerr-like black hole solution. In addition to $M$ and $a$, this spacetime has at least one more parameter: it can be seen as a deformation parameter $\epsilon$ that measures potential deviations from the Kerr geometry. This rotating black

\footnotetext{
${ }^{*}$ Corresponding author.

†Electronic address: sghosh2@jmi.ac.in, sgghosh@gmail.com

${ }_{\ddagger}^{\ddagger}$ Electronic address: hukmipankaj@gmail.com
}

hole possesses some striking properties; e.g., as the deformation parameter $\epsilon>0$, the black hole possesses two disconnected spherical horizons for a high rotation parameter and has no horizon. When $\epsilon<0$, the horizon always exists for the arbitrary $a$. Soon several researchers used a non-Kerr black hole in various astrophysical applications $17-30]$. The properties of the ergosphere and energy extraction by the Penrose process in a rotating non-Kerr black hole were investigated [30]. It turns out that for $\epsilon>0$ it has been observed that a black hole becomes more prolate than the standard Kerr black hole, whereas it is more oblate for $\epsilon<0$ [31], thereby affecting the size of the ergosphere and in turn the efficiency of the Penrose process [30].

It is rather well established that higher dimensions (HD) provide a natural playground for the string theory and they are also required for its consistency. It is interesting to study the HD extension of Einstein's theory and, in particular, its black hole solutions 32]. The HD generalization of Schwarzschild and Reissner-Nordstrom black holes were obtained by Tangherlini [33] and the rotating black hole by Myers and Perry [34]. There is a growing realization that the physics of $\mathrm{HD}$ black holes can be markedly different and much richer than in four dimensions [32, 34]. In this paper we focus our attention on the energy extraction via the Penrose process in a HD non-Kerr black hole to study the role of deformation parameter $\epsilon$ and extra dimensions in the efficiency of the Penrose process. We start with a review of HD non-Kerr solutions in Sec. II; the subsection studies the behavior of horizons and the ergosphere with respect to dimensions and the deformation parameter; Sec. III analyzes the equations of motion of particles and their motion at the equatorial plane in the vicinity of the HD non-Kerr black hole. We also obtain the negative energy states for a test particle with a specific angular momentum, orbiting around the black hole, as a function of the deformation parameter and finally we succinctly summarize our main results and evoke some perspectives to end the paper in Sec. IV. 


\section{HD NON-KERR BLACK HOLES}

The rotating black hole, in four dimension (4D) general relativity, is described by the Kerr solution [35], which is completely specified by the mass $M$ and angular momentum $a$. In 4D there is only one possible rotation axis for a rotating black hole and only one angular momentum. However, in its HD counterpart, the Myers-Perry rotating black hole, there is a multitude of angular momentum parameters, each referring to a particular rotation plane. Here, we focus on the simplest case for which there is only one angular momentum parameter, namely $a$. The rotating non-Kerr black hole metric [36] was extended to HD by Ghosh and Papnoi [37]. Beginning with a deformed HD Schwarzschild solution and applying the NewmanJanis transformation, they constructed a deformed HD Kerr-like metric with three parameters: the mass $M$, one rotation parameter $a$, and the deformation parameter $\epsilon$. The metric in $(N+3)$ dimensions for the spinning non-Kerr black hole in the standard Boyer-Lindquist-like coordinates [37] reads

$$
\begin{aligned}
d s^{2} & =g_{t t} d t^{2}+g_{r r} d r^{2}+g_{\theta \theta} d \theta^{2} \\
& +g_{\phi \phi} d \phi^{2}+2 g_{t \phi} d t d \phi+r^{2} \cos ^{2} \theta d \Omega_{N-1}^{2}
\end{aligned}
$$

with

$$
\begin{aligned}
g_{t t}= & -[1+h(r, \theta)]\left[1-\frac{\mu}{r^{N-2} \Sigma}\right], \\
g_{r r}= & \frac{[1+h(r, \theta)] \Sigma}{\Delta+a^{2} \sin ^{2} \theta h(r, \theta)}, \\
g_{\theta \theta}= & \Sigma, \\
g_{\phi \phi}= & {\left[\left(r^{2}+a^{2}+\frac{\mu}{r^{N-2} \Sigma} a^{2} \sin ^{2} \theta\right) \sin ^{2} \theta\right.} \\
& \left.+h(r, \theta) a^{2} \sin ^{4} \theta\left(1+\frac{\mu}{r^{N-2} \Sigma}\right)\right], \\
g_{t \phi}= & -[1+h(r, \theta)] \frac{\mu}{r^{N-2} \Sigma} a \sin ^{2} \theta,
\end{aligned}
$$

with

$$
\Sigma=r^{2}+a^{2} \cos ^{2} \theta \quad \Delta=r^{2}+a^{2}-\frac{\mu}{r^{N-2}} .
$$

We define the function $h(r, \theta)$ in HD as

$$
h(r, \theta)=\frac{\epsilon \mu^{3}}{8 \Sigma^{2} r^{3 N-4}},
$$

and

$$
d \Omega_{N-1}^{2}=d \chi_{1}^{2}+\sin ^{2} \chi_{1}\left(d \chi_{2}^{2}+\sin ^{2} \chi_{2}\left(\cdots d \chi_{N-1}^{2}\right)\right)
$$

is the metric of the unit $(N-1)$ sphere [38]. The metric (1) is the $\mathrm{HD}$ generalization of the Johannsen and Psaltis metric [36]. It becomes the well-known MyersPerry black hole in the limit when $h(r, \theta)$ vanishes. In general relativity, the Einstein tensor of the HD non-Kerr metric is nonzero unless $h(r, \theta)$ vanishes. Therefore, we regard the HD non-Kerr metric as a vacuum spacetime of an appropriately chosen set of modified gravity field equations that are unknown but definitely different from the Einstein equations for nonzero $h(r, \theta)$. While this does not mean that the metric does not make sense, it does, as we justify the nature of our metric, where we show that its properties are very similar to the ones of the Myers-Perry black hole and the Kerr black hole (in $4 \mathrm{D})$. In particular, we compute the location of the horizons and discuss their properties.

The definition of the function $h(r, \theta)$ in $\mathrm{HD}$ is just an extension of its 4D definition [16]. The square root of the determinant of the metric (11) reads as

$$
\sqrt{-g}=(1+h) \sqrt{\Phi} \Sigma r^{N-3} \sin \theta \cos \theta^{N-3},
$$

where $\Phi$ is the determinant of the metric (5). Here $\mu$ is an integrating constant that can be related to mass $M$ of the black hole via

$$
M=\frac{(N+1) A_{N+1} \mu}{16 \pi},
$$

$a$ is the angular momentum defined as

$$
J=\frac{A_{N+1} \mu a}{8 \pi}
$$

and $A_{N+1}$ is the area of a unit $(N+1)$ sphere given by

$$
A_{N+1}=\frac{2 \pi^{\frac{N+2}{2}}}{\Gamma\left(\frac{N+2}{2}\right)},
$$

From Eqs. (17) and (8), we get

$$
\frac{J}{M}=\frac{2 a}{N+1} .
$$

In the $4 \mathrm{D}$ limit $(\mu=2 M$ and $N=1)$, the metric (11) reduced to the non-Kerr black hole discovered in [36], and then the function $h(r, \theta)$ becomes

$$
h=\frac{\epsilon M^{3} r}{\Sigma^{2}},
$$

which is exactly the same as derived in [16, 29, 36]. Further, we discover the standard Kerr black hole in the general relativity limit $(\epsilon \rightarrow 0$ and $N \rightarrow 1)$. The standard Myers-Perry black hole 34 with a single rotation parameter is recovered for vanishing deformation parameter $\epsilon$. When the rotation parameter $a$ vanishes, one may get a deformed Schwarzschild solution [16]. It may be noted that the 4D non-Kerr metric [14] is not a solution of $R_{a b}=0$. It is kind of a perturbative way in order to include various possible deviations from the Kerr solutions in modified theories of gravity. 


\section{A. Horizons and ergosphere}

As $\epsilon=0$, the black hole is reduced to the typical Kerr black hole known in general relativity [36]. Our aim here is to discuss the effect of the extra dimension on the structure of horizons and the ergosphere. As $\epsilon=0$, the black hole is reduced to the usual Myers and Perry black hole - Similar to the Myers and Perry black hole, the above metric has two types of hypersurfaces or horizons: a stationary limit surface or infinite redshift surface and an event horizon. The static limit gets its name from the prediction that for radii smaller than the Schwarzschild radius but greater than that of the horizon, an observer cannot remain at rest and cannot stay static. It requires the prefactor of $d t^{2}$ to vanish:

$$
1-\frac{\mu}{r^{N-2} \Sigma}=0 \text { or } r^{N}+a^{2} \cos ^{2} \theta r^{N-2}-\mu=0,
$$

where we have assumed that $1+h \neq 0$ as the surface defined by $1+h=0$ is an intrinsic singularity and cannot be the infinite redshift surface [29, 30]. The surface of no return is known as the event horizon. The event horizon must satisfy $g_{t \phi}^{2}-g_{t t} g_{\phi \phi}=0$, and $\epsilon>0(<0)$ leads to more prolate (oblate) object than the $4 \mathrm{D}$ Kerr black hole [14, 29, 30]. The event horizon of the black hole is located at the outer root of the

$$
\begin{aligned}
& \Delta+a^{2} h \sin ^{2} \theta=0, \\
& \text { i.e., } r^{N}+a^{2} r^{N-2}-\mu+h a^{2} \sin ^{2} \theta r^{N-2}=0 .
\end{aligned}
$$

Clearly, the radii of the event horizon depend on $\theta$, which are different from that in the usual Kerr case, in which it is independent of $\theta$. In $4 \mathrm{D}$, for the small negative values of $\epsilon$, the spacetime has closed event horizon 29]. On the contrary, $\epsilon>0$ may lead to disconnected event horizon [29]. Thus, the stationary limit surface and event horizon depends on the spacetime dimension. However, it is seen that Eqs. (12) and (13) have at least one positive root for HD $(D \geq 6)$, i.e., just one event horizon and stationary limit surface in HD independent of the magnitude of $a$. This is a typical characteristic of the HD black hole and holds for the HD non-Kerr black hole as well. In the limit $a \rightarrow 0$, Eqs. (12) and (13) coincide with the event horizons of the nonrotating black holes [34], and they admit trivial solution $r_{+}=(\mu)^{1 / N}$.

Considering only the outer event horizon and stationary limit surface, it can be verified that the stationary limit surface always lies outside the event horizon in all dimensions. Hence, as in 4D, we call the region between the stationary limit surface and the event horizon as the ergosphere. The ergosphere is the region that lies outside of a black hole. In the ergosphere it is possible to enter and leave again, and the object moves in the direction of the spin of the black hole. It has been shown that it is possible, at least theoretically, to extract energy from the black hole in this region. The ergosphere for the 4D Kerr black hole has an oblate spherical shape. Interestingly,
TABLE I: The value of the deformation parameter at the turning point $\epsilon_{t p}$ in the HD non-Kerr black hole for different values of spin parameter $a$.

\begin{tabular}{cc|cc}
\hline \hline \multicolumn{2}{c|}{$D=4$} & \multicolumn{2}{c}{$D=5$} \\
\hline$a$ & $\epsilon_{t p}$ & $a$ & $\epsilon_{t p}$ \\
\hline 0.05 & 1044.48 & 0.075 & 123.98 \\
0.10 & 258.06 & 0.150 & 25.58 \\
0.15 & 112.44 & 0.225 & 11.06 \\
0.20 & 61.49 & 0.300 & 5.08 \\
0.25 & 37.93 & 0.375 & 2.46 \\
0.30 & 25.16 & 0.450 & 1.18 \\
0.35 & 17.479 & 0.525 & $5.40 \times 10^{-1}$ \\
0.40 & 12.51 & 0.600 & $2.18 \times 10^{-1}$ \\
0.45 & 9.14 & 0.675 & $7.23 \times 10^{-1}$ \\
0.50 & 6.75 & 0.750 & $1.64 \times 10^{-1}$ \\
\hline
\end{tabular}

the HD non-Kerr black hole may have two horizons for small values of deformation parameter $\epsilon$ even for $D \geq 6$. Thus in contrast to the Myers and Perry black hole has only one horizons for $D \geq 6$.

The non-Kerr black hole becomes more prolate than the Kerr black hole for the case $a<M$, and the size of the ergosphere increases with the increase in value of the deformation parameter $\epsilon$. We wish to bring out the effect of the extra dimension on the ergosphere. The ergospheres in various cases are shown in Figs. 1.2, which are polar plots of Eqs. (12) and (13). How the deformation parameter $\epsilon$ and $D$ affect the size of the ergosphere is demonstrated in these figures. We note that the relative shape of the ergosphere becomes more prolate, thereby increasing the area of the ergosphere with rotation parameter $a$; i.e., the faster the black hole rotates, the more the ergosphere grows. The area of ergospheres also grows with an increase in the dimension $D$. The positive value of the deformation parameter $\epsilon>0$ also facilitates the increase of the area of the ergosphere in higher dimensions, but it slightly decreases in seven dimensions (7D). However, we get disconnected horizons for the high values of $\epsilon>0$ and $a$. In Fig. 3, we plot the variation in size of the ergosphere for the negatives values of the deformation parameter $\epsilon<0$. It turns out the increase in negative values of the deformation parameter $\epsilon$ leads to shrinkage of the size of the ergosphere.

The term turning point $\left(\epsilon_{t p}\right)$ corresponding to the upper limiting value of the deformation parameter corresponds to the largest positive root of $(\partial \epsilon / \partial r)=0$ with the help of Eq. [13). Following [30], the value of the deformation parameter for the turning point $\left(\epsilon_{t p}\right)$ can be obtained from Eq. (13) as

$$
\epsilon_{t p}=-\left.\frac{8 \Delta \Sigma^{2} r^{3 N-4}}{\mu^{3} a^{2} \sin ^{2} \theta}\right|_{r=r_{t p}} .
$$

The $r_{t p}$ is the largest positive root of $(\partial \epsilon / \partial r)=0$. 


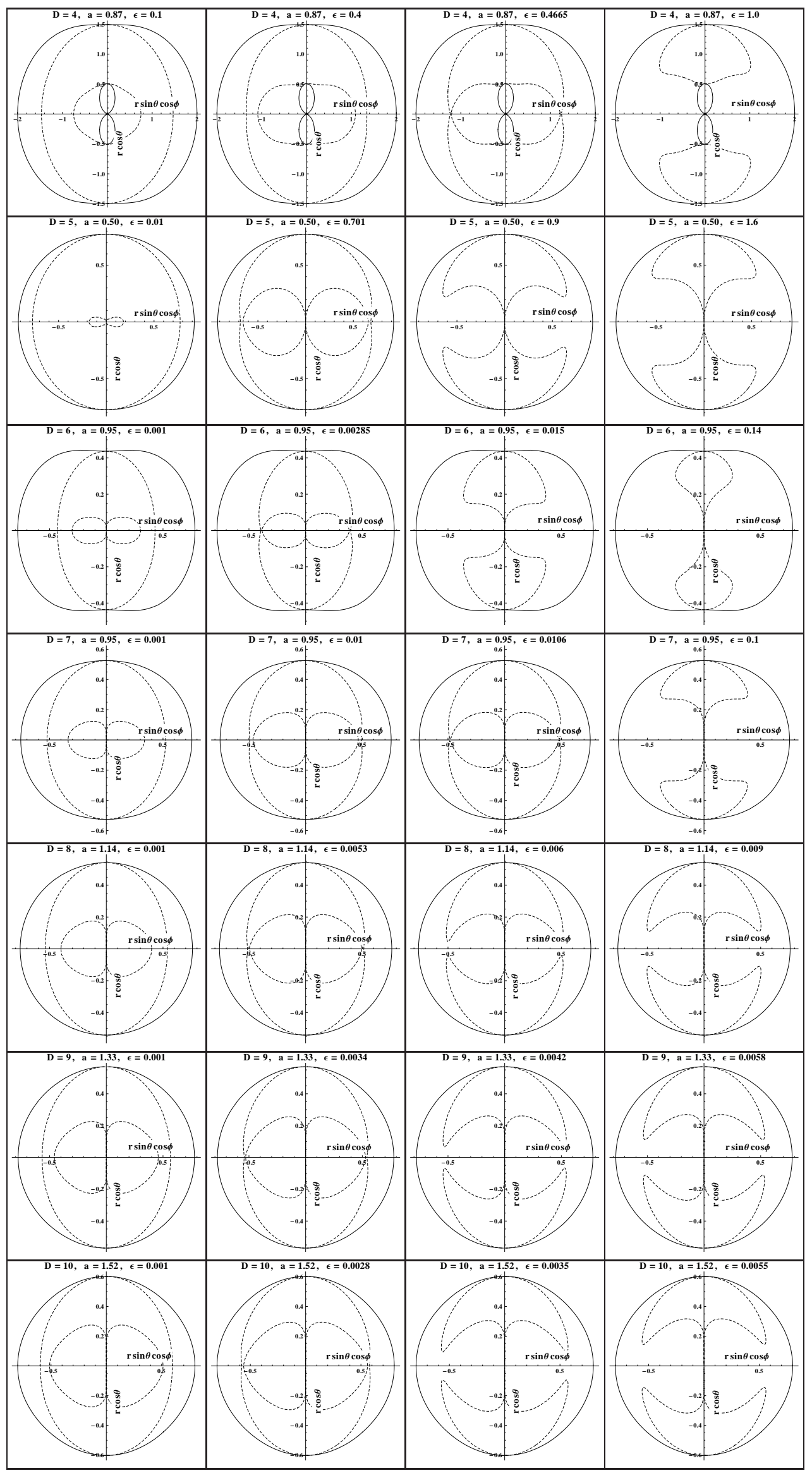

FIG. 1: The cross section of the stationary limit surface and event horizon and the variation of the ergosphere for different dimensions $(D=4, \ldots, 10)$ with deformation parameter $\epsilon$ of the HD spinning non-Kerr black hole. The increase in the value of the deformation parameter leads to a disconnected event horizon. 
The value of the deformation parameter at the intersection of the three surfaces $\Delta+a^{2} h \sin ^{2} \theta=0,1+h=0$, and $\left[1-\mu /\left(r^{N-2} \Sigma\right)\right]=0$ is denoted by $\epsilon_{i p}$. The allowed value of the deformation parameter $\epsilon$ should lie within the range $\epsilon_{i p} \leq \epsilon \leq \epsilon_{t p}$ in the ergosphere. The event horizon exists only when the deformation parameter $\epsilon$ lies between $\epsilon_{i p}$ and $\epsilon_{t p}$; when the value of the deformation parameter $\epsilon \geq \epsilon_{t p}$ and $\epsilon<\epsilon_{i p}$, no event horizon exists [30]. The value of the deformation parameter $\epsilon_{i p}$ at the intersection point remains constant in all the dimensions, i.e., $\epsilon_{i p}=-8$, whereas from Tables 1 and II we conclude that the value of the deformation parameter at the turning point $\epsilon_{t p}$ decreases as the value of the spin parameter $a$ increases in each dimension.

TABLE II: The value of the deformation parameter at the turning point $\epsilon_{t p}$ in the HD non-Kerr black hole for different values of spin parameter $a$.

\begin{tabular}{cc|cc}
\hline \hline \multicolumn{3}{c|}{$D=6$} & \multicolumn{2}{c}{$\mathrm{D}=7$} \\
\hline$a$ & $\epsilon_{t p}$ & $a$ & $\epsilon_{t p}$ \\
\hline 0.10 & 54.01 & 0.125 & 33.12 \\
0.20 & 11.46 & 0.250 & 6.50 \\
0.30 & 3.86 & 0.375 & 1.94 \\
0.40 & 1.46 & 0.500 & $6.33 \times 10^{-1}$ \\
0.50 & $5.53 \times 10^{-1}$ & 0.625 & $2.07 \times 10^{-1}$ \\
0.60 & $1.98 \times 10^{-1}$ & 0.750 & $6.64 \times 10^{-2}$ \\
0.70 & $6.58 \times 10^{-2}$ & 0.875 & $2.11 \times 10^{-2}$ \\
0.80 & $1.99 \times 10^{-2}$ & 1.000 & $6.79 \times 10^{-3}$ \\
0.90 & $5.57 \times 10^{-3}$ & 1.125 & $2.25 \times 10^{-3}$ \\
1.0 & $1.47 \times 10^{-3}$ & 1.250 & $7.79 \times 10^{-4}$ \\
\hline
\end{tabular}

We shall next show how this ergosphere can be used to extract energy from the black hole, i.e., by throwing in particles with suitable parameters, so they attain negative energy relative to an asymptotic observer. We also explicitly study the effect of extra dimensions and the deformation parameter on the energy extraction process.

\section{ENERGY EXTRACTION FROM HD BLACK HOLE}

The Penrose process theoretically suggested by Penrose [3] is that the energy can be extracted from a spinning black hole. This is made possible because of the existence of the ergosphere, where it is possible to have timelike or a null trajectory with negative energy. Penrose considered an infalling particle disintegration in the ergosphere of a Kerr black hole. One of the particles produced in this process might be thrown into a negative energy (with respect to infinity) orbit, while the other one will have an energy larger than that of the infalling one. The particle with a negative energy will be swallowed by a black hole while the other one will escape to infinity with a net gain in energy. The energy excess arises eventually from the rotational energy of the black hole. Here we apply the Penrose process to the spinning HD non-Kerr black hole. We now consider the trajectory of such a negative energy particle in $D$ dimensions. The equation of motion of such a particle can be derived from the Lagrangian $\mathcal{L}$,

$$
\mathcal{L}=\frac{m}{2} g_{i j} \dot{x}^{i} \dot{x}^{j}
$$

where an overdot denotes the derivative with respect to affine parameter $\tau / m$ ( $\tau$ being the proper time). Since the metric (11) is stationary and axis symmetric, the motion of a test particle with $D$-momentum $p^{i}$ is described by its rest mass $m$, the total energy $E$ (as measured from $\infty)$ is $\partial \mathcal{L} / \partial \dot{t}=p_{t}=-m E$, and the component of angular momentum is $\partial \mathcal{L} / \partial \dot{\phi}=p_{\phi}=m L$. These expressions on using the metric (11) become

$$
m E=[1+h]\left[\left(1-\frac{\mu}{r^{N-2 \Sigma}}\right) \dot{t}+\frac{\mu}{r^{N-2} \Sigma} a \sin ^{2} \theta \dot{\phi}\right]
$$

and

$$
\begin{aligned}
m L= & -[1+h] \frac{\mu}{r^{N-2} \Sigma} a \sin ^{2} \theta \dot{t} \\
+ & {\left[\left(r^{2}+a^{2}+\frac{\mu}{r^{N-2} \Sigma} a^{2} \sin ^{2} \theta\right) \sin ^{2} \theta\right.} \\
& \left.+h a^{2} \sin ^{4} \theta\left(1+\frac{\mu}{r^{N-2} \Sigma}\right)\right] \dot{\phi} .
\end{aligned}
$$

The conservation equation for the particles rest mass $p^{j} p_{j}=-m^{2}$ gives

$$
\begin{aligned}
& g_{\phi \phi} E^{2}+2 g_{t \phi} E L+g_{t t} L^{2}+\psi\left(g^{r r} p_{r}^{2}+g^{\theta \theta} p_{\theta}^{2}+g_{\chi_{1} \chi_{1}} p_{\chi_{1}}^{2}\right. \\
& \left.+g^{\chi_{2} \chi_{2}} p \chi_{\chi_{2}}^{2}+\cdots+g^{\chi_{N-1} \chi_{N-1}} \chi_{\chi_{N-1}}^{2}+m^{2}\right)=0 .
\end{aligned}
$$

If the particle is constrained on the equatorial plane $\theta=$ $\pi / 2$, then $p_{\theta}=p_{\chi_{1}}=p_{\chi_{2}}=\cdots=0$. Equation (18) can be rewritten as

$$
\alpha E^{2}-2 \beta E+\gamma+\delta p_{r}^{2}=0
$$

with

$$
\begin{aligned}
& \alpha=\frac{1}{\psi}\left(r^{2}+a^{2}+\frac{a^{2} h}{r^{2}}\left(r^{2}+\frac{\mu}{r^{N-2}}\right)+\frac{\mu a^{2}}{r^{N}}\right) \\
& \beta=\frac{L}{\psi}(1+h) \frac{\mu a}{r^{N}} \\
& \gamma=\frac{L^{2}}{\psi}(1+h)\left(1-\frac{\mu}{r^{N}}\right)-m^{2} \\
& \delta=-\frac{r^{2}(1+h)}{\Delta+a^{2} h} p_{r}^{2} .
\end{aligned}
$$

where $\psi=(1+h)\left(\Delta+a^{2} h\right)<0$ for $r>r_{+}$(outer horizon). It is easy to check that $\psi=0 \Leftrightarrow \Delta+a^{2} h \sin ^{2} \theta=0$. 


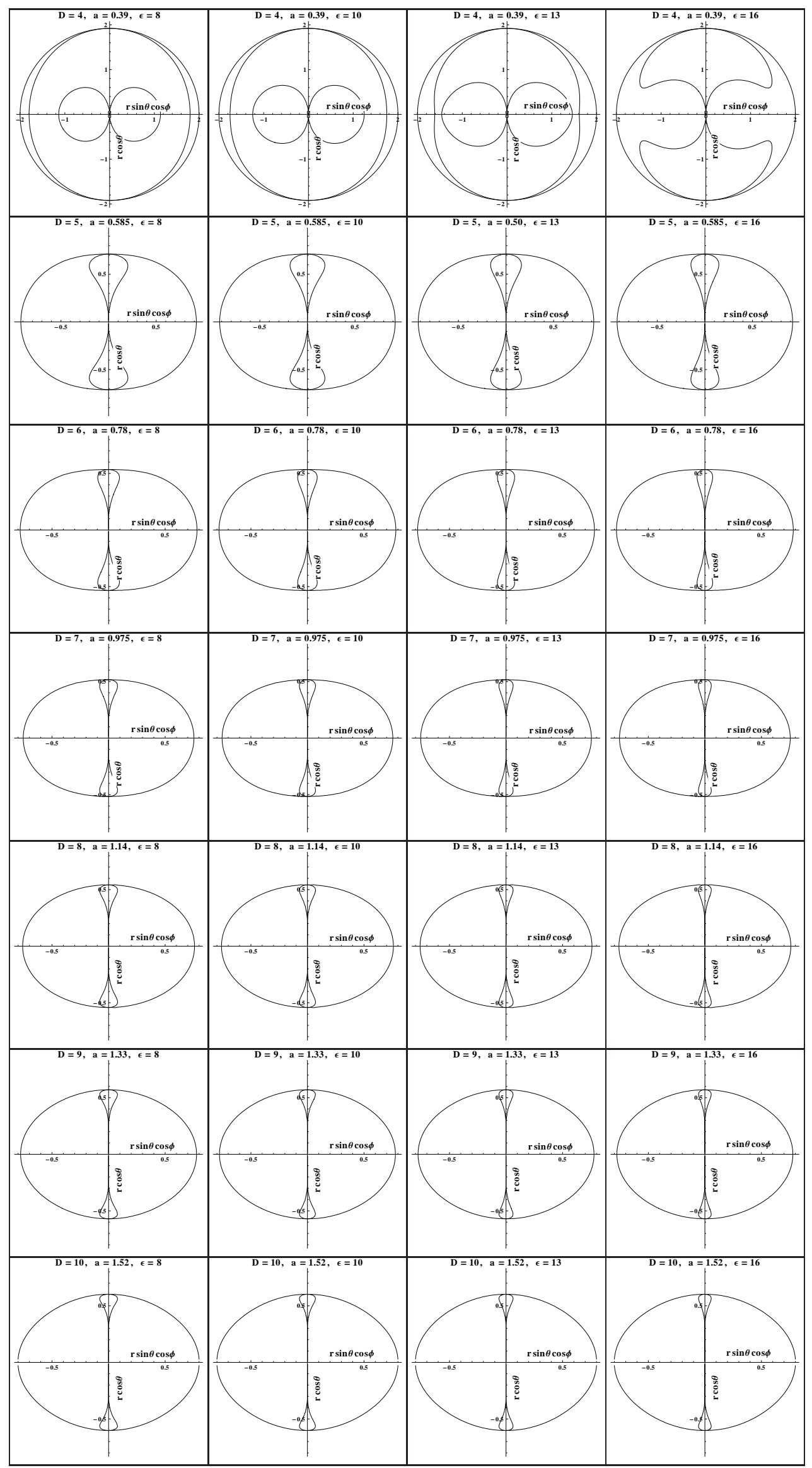

FIG. 2: The cross section of the stationary limit surface and event horizon and the variation of the ergosphere for different dimensions $(D=4, \ldots, 10)$ with four different values of deformation parameter $\epsilon$. It shows a disconnected event horizon for higher values of deformation parameter $\epsilon>0$. 


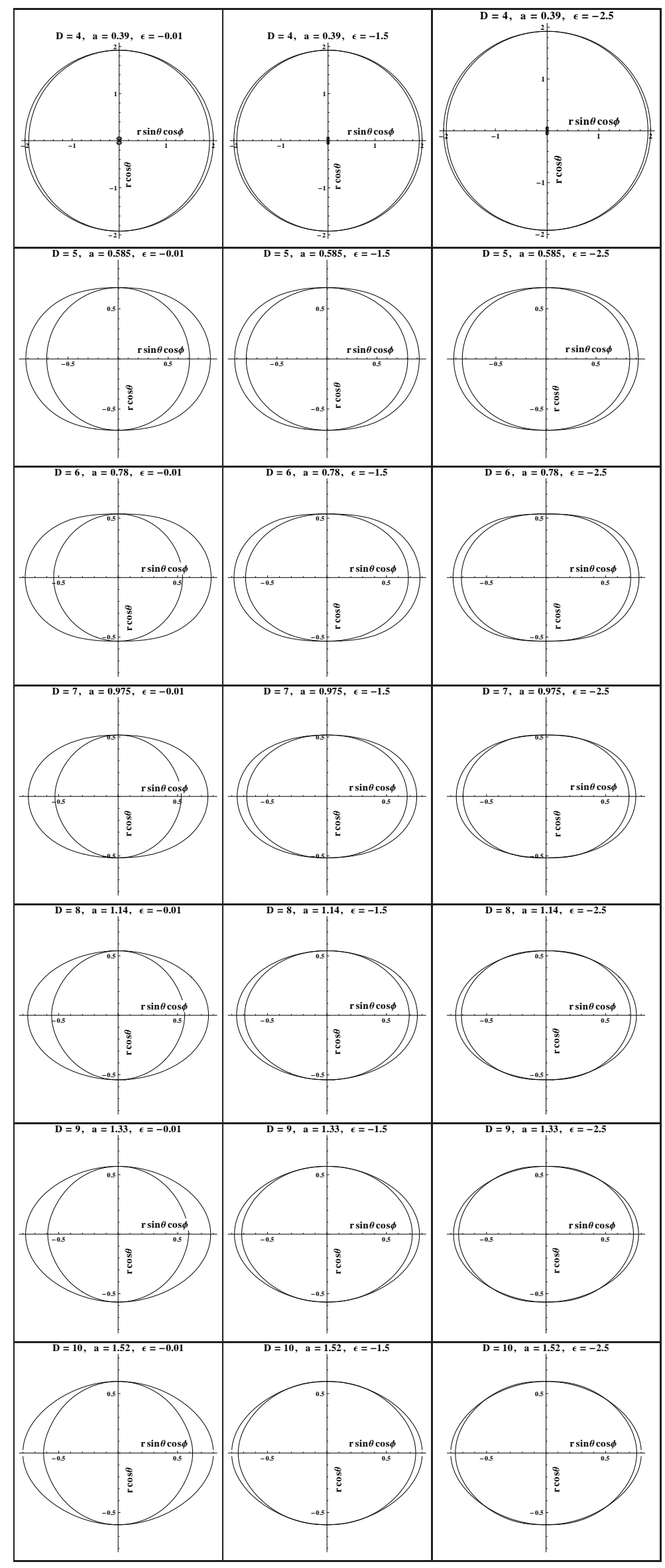

FIG. 3: The cross section of the stationary limit surface and the event horizon and the variation of the ergosphere for different dimensions $(D=4, \ldots, 10)$ with negative values of deformation parameter $\epsilon$. For $\epsilon<0$ the ergosphere region shrinks with an increase in the magnitude of $\epsilon$. 


\section{A. Negative energy states in the Penrose process}

In the Penrose process, we are interested in the region of spacetime over which energy is negative and the orbit of particles with negative energy in the ergosphere is very important to extract energy from the black hole. As in the 4D Kerr, the negative energy states occur due to counterrotating orbits. Unlike 4D Kerr or non-Kerr black holes, where the black hole has two horizons, the HD non-Kerr black hole has just one horizon where the 4-velocity of the counterrotating observer tends to zero. In the Penrose process [3], a particle falling onto a black hole splits up into two particles at some $r>r_{+}$. The one particle falling into a black hole has a negative energy (relative to $\infty$ ), and hence, the outgoing particle leaving the ergosphere has more energy than the incident particle; thus energy is extracted. In fact, for the $D$-momentum $p_{i}=m u_{i}$, the energy $E=-p^{i} \xi_{i}$ may not be positive in the ergosphere; hence, one can extract energy from the black hole by absorbing a particle with negative energy [3]. Now, we shall focus on the effect of the deformation parameter and extra dimension on the region of the negative energy state for the HD non-Kerr black hole. In the HD non-Kerr black hole the orbit of the particle with the negative energy obeys $\alpha>0, \beta<0$, $\gamma>\delta$.

In Figs. 4 and 5, we demonstrate that the negative energy states near the horizon with different values of the deformation parameter in different dimensions can be achieved if $L a<0$. It is interesting to note that the negative energy $E$ is sensitive to both deformation parameter $\epsilon$ and extra dimension $D$. We see that the extra dimension and deformation parameter $\epsilon$ favors negative energy states; i.e., the negative energy $E$ increases with both an increase in deformation parameter $\epsilon$ and extra dimension $D$.

\section{B. Efficiency of Penrose process}

One of the most interesting processes for extracting energy from a rotating black hole is the Penrose process. As mentioned in the Introduction, in the original Penrose paper [3], we would have to assume that the incoming particle in the ergosphere may be decomposed into two subparticles and one of them with negative energy will fall into the black hole, while the other is ejected to the exterior of the ergosphere and will have more energy than original particle [4 8]. Here we apply the recipe of energy extraction proposed in [3] to the deformed HD Kerr black hole and use the recipe provided by Bhat et al. [5]. In the energy extraction process, we take the incident particle with the $D$-momentum $p_{i}^{(i n)}$, which breaks up into two particles $p_{i}^{(b h)}$ and $p_{i}^{(\text {out })}$ with the $D$-momentum in the ergosphere. We take the total $D$-momentum as conserved at the point of break, which reads as $p_{i}^{(i n)}=p_{i}^{(b h)}+p_{i}^{(\text {out })}$. Here the momentum of the particles is non-spacelike and therefore lies inside the light cone. To discuss the particles' energy, we consider the timelike killing vector

$$
\begin{aligned}
\xi^{(t) i}= & (1,0,0, \ldots, 0), \quad g_{i j} \xi^{(t) i} \xi^{(t) j}=g_{t t}<0, \\
& p_{i}^{(i n)} \xi^{(t) i}=E^{(i n)}=E^{(b h)}+E^{(\text {out })} .
\end{aligned}
$$

Inside the ergosphere, the killing vector $\xi^{(t) i}$ becomes spacelike vector and $g_{t t}>0$. Hence, $E^{(b h)}=p_{i}^{(b h)} \xi^{(t) i}$ can possibly be negative. Thus

$$
E^{(\text {out })}=E^{(i n)}-E^{(b h)}>E^{(i n)} .
$$

and hence, we may say that the Penrose process extracts the rotational energy of the black hole.

We get the best result in the energy extraction process by choosing the rest mass $m^{(i n)}$ and energy $E^{(i n)}$ of an incident particle equal to unity, which splits into two particles; the particle $(b h)$ absorbed by the HD non-Kerr black hole has $\Omega \rightarrow \Omega^{-}$and the particle (out) escaping to infinity has $\Omega \rightarrow \Omega^{+}$. From equation conservation of the energy and momentum

$$
\begin{aligned}
& E^{(\text {in })}=E^{(\text {out })}+E^{(b h)}, \\
& L^{(\text {in })}=L^{(\text {out })}+L^{(b h)} .
\end{aligned}
$$

The particles falling into the black hole have energy $E^{(b h)}$ and angular momentum $L^{(b h)}$. The particle falling into the black hole has negative energy, and hence the outgoing particle, leaving the ergosphere, has more energy than the incident particle. The maximum efficiency is obtained if we take the radial velocity component of velocities to be zero, at the point of the split.

Here, we are interested in the contribution of both the deformation parameter and the extra dimension on the efficiency of the Penrose process for which we again rely on the prescription given in [5]. For this purpose, according to the conservation law of angular momentum, we have

$$
U^{(i n)}=m^{(b h)} U^{(b h)}+m^{(o u t)} U^{(o u t)}
$$

where $U_{i}^{(I)}(I=i n, b h$, out $)$ denote the $D$-velocity of the particle at the point of the split, which can be expressed as

$$
U_{i}^{(I)}=u_{t}\left(1,0,0, \Omega^{(I)}, 0, \ldots, 0\right)
$$

where

$$
u_{t}=\frac{E}{g_{t t}+g_{t \phi} \Omega^{I}},
$$

and 

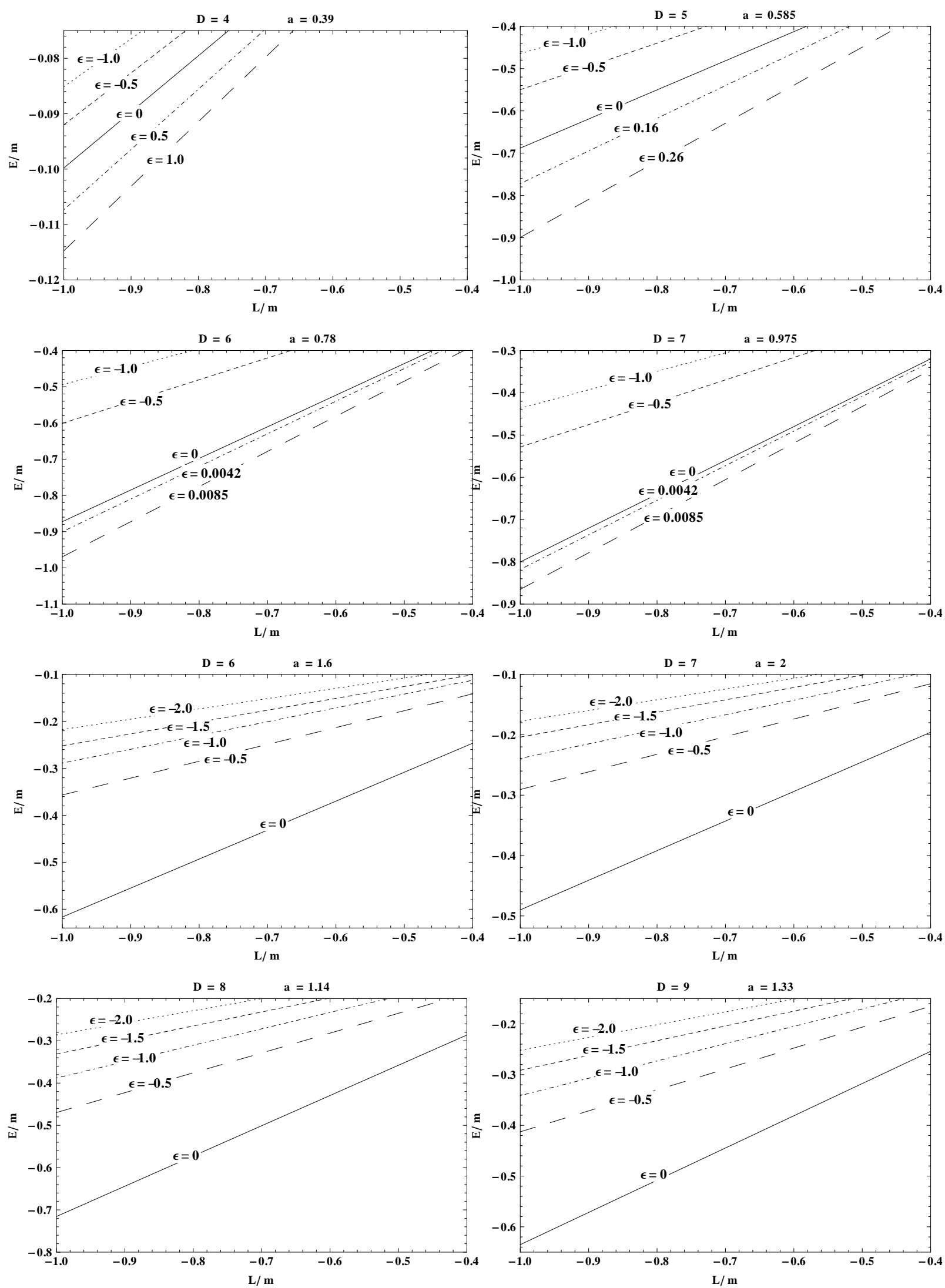

FIG. 4: The negative energy state $E$ allowed for the angular momentum $L$ and rest mass $m$ for the particle in the different dimensions $(D=4, \ldots, 9)$ with different values of deformation parameter $\epsilon$ near the event horizon inside the ergosphere. 


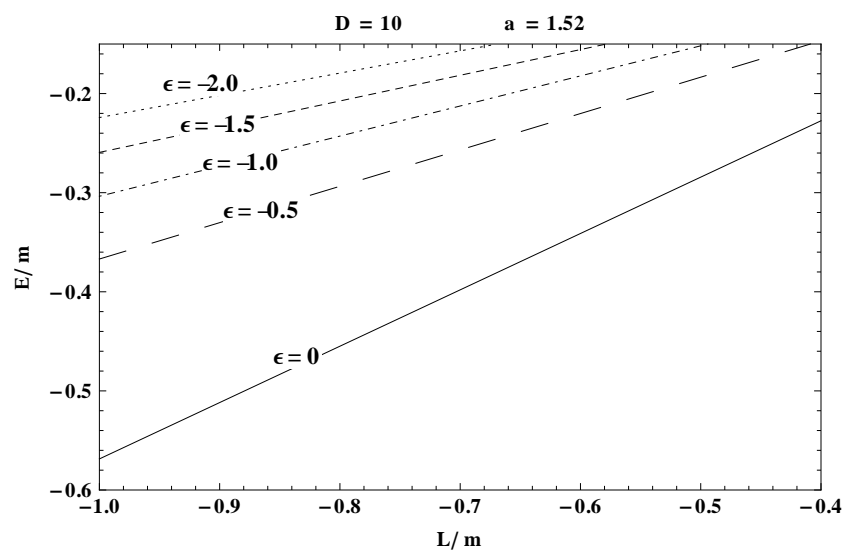

FIG. 5: The negative energy state $E$ allowed for the angular momentum $L$ and rest mass $m$ for the particle in the dimension $(D=10)$ with different values of deformation parameter $\epsilon$ near the event horizon inside the ergosphere.

$$
\Omega^{(I)}=\frac{a \mu(1+h)\left[h\left(\mu-r^{N}\right)+\mu\right] \sqrt{-r^{2 N}(1+h)\left[h r^{N}-(1+h) \mu\right]\left[a^{2}(1+h) r^{N}+r^{2}\left(r^{N}-\mu\right)\right]}}{a^{2}(1+h)\left[(1+h) \mu^{2}+r^{2 N}+\mu r^{N}\right]+r^{N+2}} .
$$

In the general relativity limit, $h \rightarrow 0, N \rightarrow 1$, Eq. (30) becomes

$$
\Omega^{(I)}=\frac{\sqrt{2} r^{2} \sqrt{\frac{M}{r}\left(r^{2}-2 M r+a^{2}\right)}+4 a M^{2}}{a^{2}\left(r^{2}+2 M r+4 M^{2}\right)+r^{4}},
$$

Here $\Omega^{(I)}$ is the asymptotic angular velocity of the $i$ th particle. The angular velocity of particles after the split always lies within the future directed light cone and hence is constrained in between $\Omega^{-}<\Omega^{I}<\Omega^{+}$

,where

$$
\Omega_{ \pm}=\frac{-g_{t \phi} \pm \sqrt{-\psi}}{g_{\phi \phi}}=\frac{a \mu(1+h) \pm \sqrt{(1+h)\left[r^{2 N} a^{2}(1+h)+r^{N+2}\left(r^{N}-\mu\right)\right]}}{a^{2} \mu(1+h)+a^{2} r^{N}(1+h)+a^{2} r^{N+2}(1+h)} .
$$

In the general relativity limit, $h \rightarrow 0, N \rightarrow 1$, Eq. (32) reads as

$$
\Omega_{ \pm}=\frac{2 a M \pm \sqrt{(a r)^{2}+r^{3}(-2 M+r)}}{r^{3}+a^{2}(2 M+r)}
$$

It turns out that the maximal output will be gained as $\Omega^{(b h)} \rightarrow \Omega^{-}$and $\Omega^{(\text {out })} \rightarrow \Omega^{+}$.

The important question in the black hole energy extraction process is the efficiency of the process. It is supposed to be one of the many important parameters in active galactic nuclei. Hence, it is very relevant to examine the efficiency of the Penrose process. In Fig. 6. we plot the maximum efficiency $\eta_{\max }$ versus the deformation parameter $\epsilon$. From the figure we conclude that as the deformation parameter increases from $\epsilon<0$ to $\epsilon>0$ the maximum efficiency $\eta_{\max }$ of the energy extraction process increases with the dimensions. We further see as the dimension increases the maximum efficiency increases for the same value of the spin parameter. In Tables III VI, we show the variation of maximum efficiency $\eta_{\max }$ corresponding to the different values of deformation parameter $\epsilon$ and the spin parameter $a$. The value of the maximum efficiency increases in 4D for the increase in the value of deformation parameter $\epsilon$ (Table【II). Similarly, the maximum efficiency also increases with the increase in the spin 

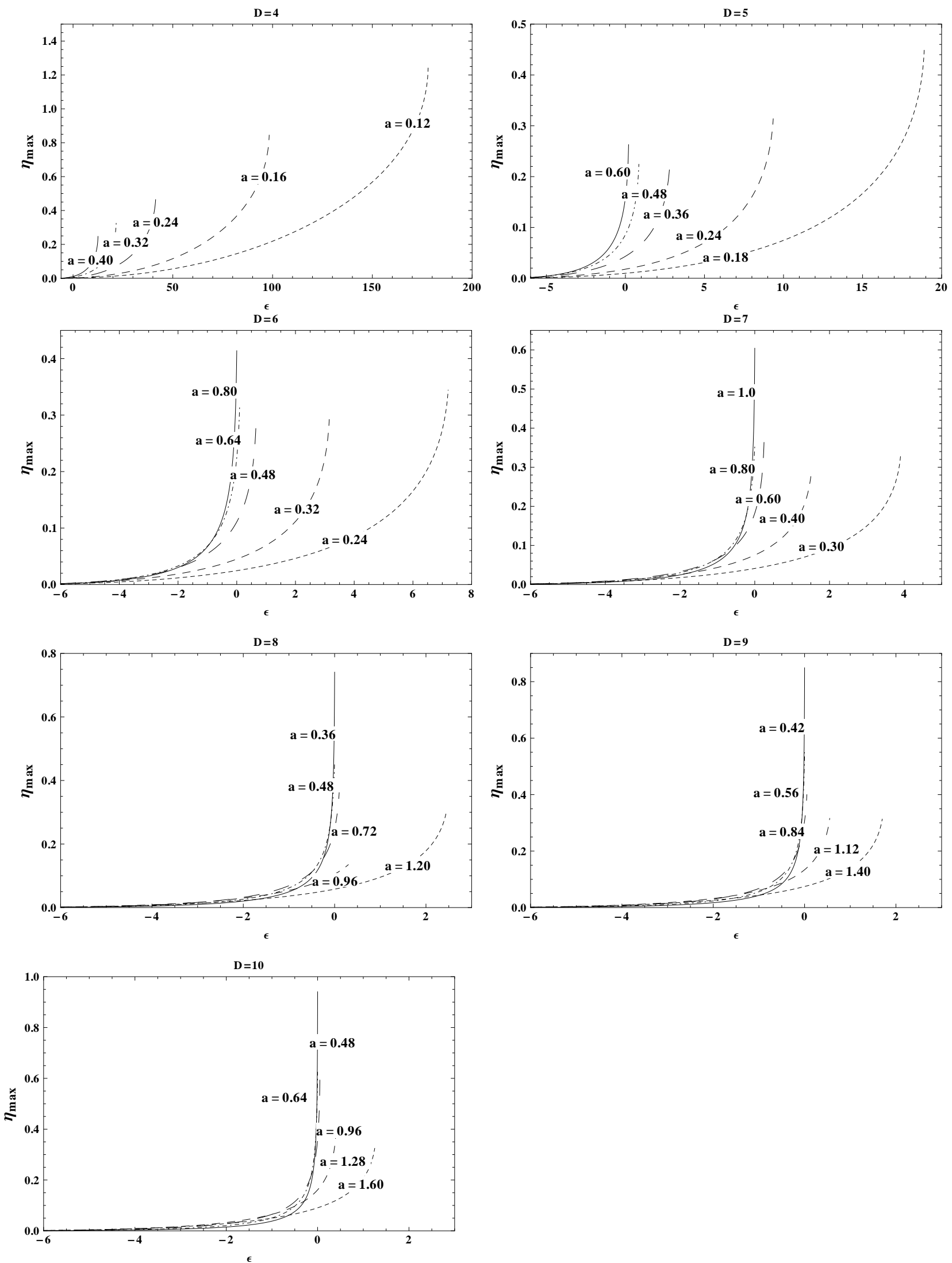

FIG. 6: The variation of the maximum efficiency of the energy extraction process for different dimensions $(D=4, \ldots, 10)$ with the deformation parameter $\epsilon$. 
parameter $a$ for the same value of deformation parameter $\epsilon$. Also we see that the value of the maximum efficiency first increases with the increase in value spin parameter $a$ and then starts decreasing as the value of the spin parameter $a>1$ (Tables IV VI). Further, we also conclude that as the value of the spin parameter $a>0.75$ we get the maximum efficiency $\eta_{\max }$ only for the values of the deformation parameter $\epsilon \leq 0$.

Defining then the efficiency $\eta$ of the process as a gain in energy per input of energy, i.e.,

$$
\eta=\frac{m^{(\text {out })} E^{(\text {out })}-E^{(\text {in })}}{E^{(\text {in })}}=m^{(\text {out })} \frac{E^{(\text {out })}}{E^{(i n)}}-1 .
$$

Now, using momentum conservation Eq. (27), we find that

$$
m^{(\text {out })} \frac{E^{(\text {out })}}{E^{(\text {in })}}=\frac{\left(\Omega^{(i n)}-\Omega^{-}\right)\left(1-\frac{\mu}{r^{N}}\left(1+\Omega^{+} a\right)\right)}{\left(\Omega^{+}-\Omega^{-}\right)\left(1-\frac{\mu}{r^{N}}\left(1+\Omega^{(i n)} a\right)\right)}
$$

Now in the limit when the split tends to $r_{+}$,

$$
\begin{aligned}
\eta_{\max } & =\left.\frac{\sqrt{1+g_{t t}}-1}{2}\right|_{r=r_{+}} \\
& =\frac{1}{2}\left[\left(\frac{\mu}{r^{N}}\right)^{\frac{1}{2}} \sqrt{1-\frac{\epsilon \mu^{2}}{8 r^{2 N}}\left(1+\frac{\mu}{r^{N}}\right)}-1\right]_{r=r_{+}}
\end{aligned}
$$

It is known that $\eta_{\max } \sim 20.7 \%$ for the extreme Kerr black hole [5], which is amplified to $60 \%$ for the deformed Kerr black hole. However, there is no upper limit on $\eta$ in HD.

\section{CONCLUSIONS}

The non-Kerr black hole solution has an additional deformation parameter $\epsilon$ than the Kerr black hole, and it produces deviation from Kerr geometry but with a richer configuration of the ergosphere. This motivates us to reconsider the Penrose process in the non-Kerr black hole scenario as energy is extracted from the ergosphere.
We have discussed the energy extraction via the Penrose process from HD non-Kerr black holes. However, there are problems with this method as well. Penrose himself said that the method is inefficient [3], although later [2] he showed that the theoretical efficiency could reach $20 \%$ extra energy up to $60 \%$. We have studied in detail the influence of the deformation parameter $\epsilon$ and extra dimensions on the structure of horizons and the ergosphere of the Kerr black hole. We can conclude that energy extraction via the Penrose process is more realistic in the HD non-Kerr black hole as its efficiency is enhanced in the HD non-Kerr case. The presence of the deformation parameter and extra dimension influence the behavior of horizons, ergospheres, and negative energy states. It is interesting to see that the HD non-Kerr black hole $(D \geq 6)$ can have two horizons for small values of deformation parameters $\epsilon$, whereas the Myers-Perry black hole $(D \geq 6)$ has just one horizon. The higher values of $\epsilon>0$ is not viable as it leads to disconnected horizons (Figs. 1 12 ). We have also calculated the condition on $\epsilon$ for the proper horizons in the HD non-Kerr black hole. It has been demonstrated that the Penrose process is more efficient than the Myers-Perry black hole or the HD Kerr black hole $(\epsilon=0)$ with no upper bound in the efficiency (Tables IV V] and VI) in contrast to the 4D Kerr black hole where the maximum efficiency is just 20\%. Further, it is seen that the ergosphere is sensitive to both deformation parameter $\epsilon$ and extra dimension; both of them individually lead the enlargement of negative energy states and also facilitate the Penrose process by enhancing the efficiency of the energy extraction process. However, in the presence of both, i.e., in the HD non-Kerr black hole, we expect only a tiny gain in efficiency of the Penrose process.

\section{Acknowledgments}

S. G. G. and P. S. thank IUCAA, Pune, for kind hospitality where a part of this work was done, and one of the authors (S. G. G.) also thanks the University Grant Commission (UGC) for the major research project grant F. NO. 39-459/2010 (SR).
[1] D. L. Meier, S. Koide, and Y. Uchida, Science 291, 84 (2001).

[2] S. Chandrasekhar, The Mathematical Theory of Black Holes (Oxford University Press, Oxford, 1983).

[3] R. Penrose, Riv. Nuovo Cimento Numero Speciale 1, 252 (1969)[Gen. Relativ. Gravit. 34, 1141 (2002)].

[4] J. M. Bardeen, W. H. Press, and S. A. Teukolsky, Astrophys. J. 178, 347 (1972).

[5] M. Bhat, S. Dhurandrhar, and N. Dadhich, J. Astrophys. Astron. 6, 85 (1985).

[6] D. Christodoulou, Phys. Rev. Lett. 25, 1596 (1970).

[7] S. Parthasarathy, S. M. Wagh, S. V. Dhurandhar, and N.
Dadhich, Astrophys. J. 307, 38 (1986).

[8] R. Penrose and R. M. Floyd, Nat. Phys. Sci. 229, 117 (1971).

[9] K. Prabhu and N. Dadhich, Phys. Rev. D 81, 024011 (2010).

[10] M. Nozawa and K. Maeda, Phys. Rev. D 71, 084028 (2005).

[11] A. A. Abdujabbarov and B. J. Ahmedov, Phys. Rev. D 84, 044044 (2011).

[12] A. A. Abdujabbarov, B. J. Ahmedov, S. R. Shaymatov, and A. S. Rakhmatov, Astophys. Space Sci. 334, 237 (2011). 
TABLE III: The maximum energy efficiency $\eta_{\max }$ of energy extraction in the HD non-Kerr black hole for different values of deformation parameter $\epsilon$ corresponding to dimension $D=4$.

\begin{tabular}{cccccccccccc}
\hline \hline$\epsilon$ & $\mathrm{a}=0.1 \mathrm{a}=0.2 \mathrm{a}=0.3$ & $\mathrm{a}=0.4$ & $\mathrm{a}=0.5$ & $\mathrm{a}=0.6$ & $\mathrm{a}=0.7$ & $\mathrm{a}=0.8$ & $\mathrm{a}=0.9$ & $\mathrm{a}=1.0$ & $\mathrm{a}=1.1$ \\
\hline-0.5 & $0.055 \%$ & $0.222 \%$ & $0.509 \%$ & $0.926 \%$ & $1.493 \%$ & $2.237 \%$ & $3.197 \%$ & $4.424 \%$ & $5.972 \%$ & $7.838 \%$ & $9.756 \%$ \\
-0.4 & $0.056 \%$ & $0.228 \%$ & $0.524 \%$ & $0.955 \%$ & $1.545 \%$ & $2.324 \%$ & $3.344 \%$ & $4.674 \%$ & $6.416 \%$ & $8.643 \%$ & $11.099 \%$ \\
-0.3 & $0.058 \%$ & $0.235 \%$ & $0.539 \%$ & $0.985 \%$ & $1.597 \%$ & $2.414 \%$ & $3.497 \%$ & $4.944 \%$ & $6.919 \%$ & $9.650 \%$ & $13.002 \%$ \\
-0.2 & $0.059 \%$ & $0.241 \%$ & $0.554 \%$ & $1.015 \%$ & $1.651 \%$ & $2.508 \%$ & $3.659 \%$ & $5.236 \%$ & $7.498 \%$ & $10.996 \%$ & $16.212 \%$ \\
-0.1 & $0.061 \%$ & $0.247 \%$ & $0.570 \%$ & $1.046 \%$ & $1.707 \%$ & $2.604 \%$ & $3.829 \%$ & $5.554 \%$ & $8.180 \%$ & $13.061 \%$ & $24.877 \%$ \\
0 & $0.062 \%$ & $0.254 \%$ & $0.585 \%$ & $1.077 \%$ & $1.764 \%$ & $2.704 \%$ & $4.008 \%$ & $5.901 \%$ & $9.009 \%$ & $20.710 \%$ & \\
0.1 & $0.064 \%$ & $0.261 \%$ & $0.602 \%$ & $1.109 \%$ & $1.822 \%$ & $2.808 \%$ & $4.197 \%$ & $6.284 \%$ & $10.072 \%$ & & \\
0.2 & $0.066 \%$ & $0.267 \%$ & $0.618 \%$ & $1.141 \%$ & $1.881 \%$ & $2.915 \%$ & $4.397 \%$ & $6.709 \%$ & $11.580 \%$ & & \\
0.3 & $0.067 \%$ & $0.274 \%$ & $0.635 \%$ & $1.175 \%$ & $1.943 \%$ & $3.026 \%$ & $4.609 \%$ & $7.187 \%$ & $14.593 \%$ & & \\
0.4 & $0.069 \%$ & $0.281 \%$ & $0.652 \%$ & $1.209 \%$ & $2.005 \%$ & $3.141 \%$ & $4.835 \%$ & $7.732 \%$ & & & \\
0.5 & $0.071 \%$ & $0.288 \%$ & $0.669 \%$ & $1.243 \%$ & $2.070 \%$ & $3.261 \%$ & $5.073 \%$ & $8.366 \%$ & & & \\
\hline
\end{tabular}

TABLE IV: The maximum energy efficiency $\eta_{\max }$ of energy extraction in the HD non-Kerr black hole for different values of deformation parameter $\epsilon$ corresponding to dimension $D=5$.

\begin{tabular}{|c|c|c|c|c|c|c|c|c|c|c|}
\hline $\bar{\epsilon}$ & $a=0.15$ & $a=0.30$ & $\mathrm{a}=0.45$ & $\mathrm{a}=0.6 \quad \mathrm{a}=0.75$ & $a=0.9$ & $\mathrm{a}=1.05$ & $a=1.2$ & $\mathrm{a}=1.35$ & $a=1.5$ & $\mathrm{a}=1.65$ \\
\hline$\overline{-0.5}$ & $0.587 \%$ & $2.400 \%$ & $5.457 \%$ & $\begin{array}{ll}9.071 \% & 11.289 \%\end{array}$ & $11.345 \%$ & $10.295 \%$ & $8.985 \%$ & $7.747 \%$ & $6.672 \%$ & $5.769 \% \%$ \\
\hline-0.4 & $0.604 \%$ & $2.491 \%$ & $5.772 \%$ & $9.937 \% \quad 12.814 \%$ & $13.107 \%$ & $11.978 \%$ & $10.489 \%$ & $9.062 \%$ & $7.816 \%$ & $6.765 \%$ \\
\hline-0.3 & $0.622 \%$ & $2.584 \%$ & $6.111 \%$ & $10.963 \% 14.819 \%$ & $15.528 \%$ & $14.323 \%$ & $12.597 \%$ & $10.912 \%$ & $9.431 \%$ & $8.175 \%$ \\
\hline-0.2 & $0.639 \%$ & $2.680 \%$ & $6.476 \%$ & $12.209 \% 17.668 \%$ & $19.214 \%$ & $17.961 \%$ & $15.893 \%$ & $13.820 \%$ & $11.978 \%$ & $10.407 \%$ \\
\hline-0.1 & $0.658 \%$ & $2.797 \%$ & $6.870 \%$ & $13.780 \% 22.390 \%$ & $26.197 \%$ & $25.079 \%$ & $22.417 \%$ & $19.618 \%$ & $17.089 \%$ & $14.910 \%$ \\
\hline 0 & $0.676 \%$ & $2.882 \%$ & $7.299 \%$ & $15.887 \% 36.089 \%$ & $183.785 \%$ & & & & & \\
\hline 0.1 & $0.694 \%$ & $2.987 \%$ & $7.768 \%$ & $19.073 \%$ & & & & & & \\
\hline 0.2 & $0.713 \%$ & $3.096 \%$ & $8.282 \%$ & $26.289 \%$ & & & & & & \\
\hline 0.3 & $0.733 \%$ & $3.208 \%$ & $8.850 \%$ & & & & & & & \\
\hline 0.4 & $0.752 \%$ & $3.324 \%$ & $9.484 \%$ & & & & & & & \\
\hline 0.5 & $0.772 \%$ & $3.444 \%$ & $10.198 \%$ & & & & & & & \\
\hline
\end{tabular}

TABLE V: The maximum energy efficiency $\eta_{\max }$ of energy extraction in the HD non-Kerr black hole for different values of deformation parameter $\epsilon$ corresponding to dimension $D=6$.

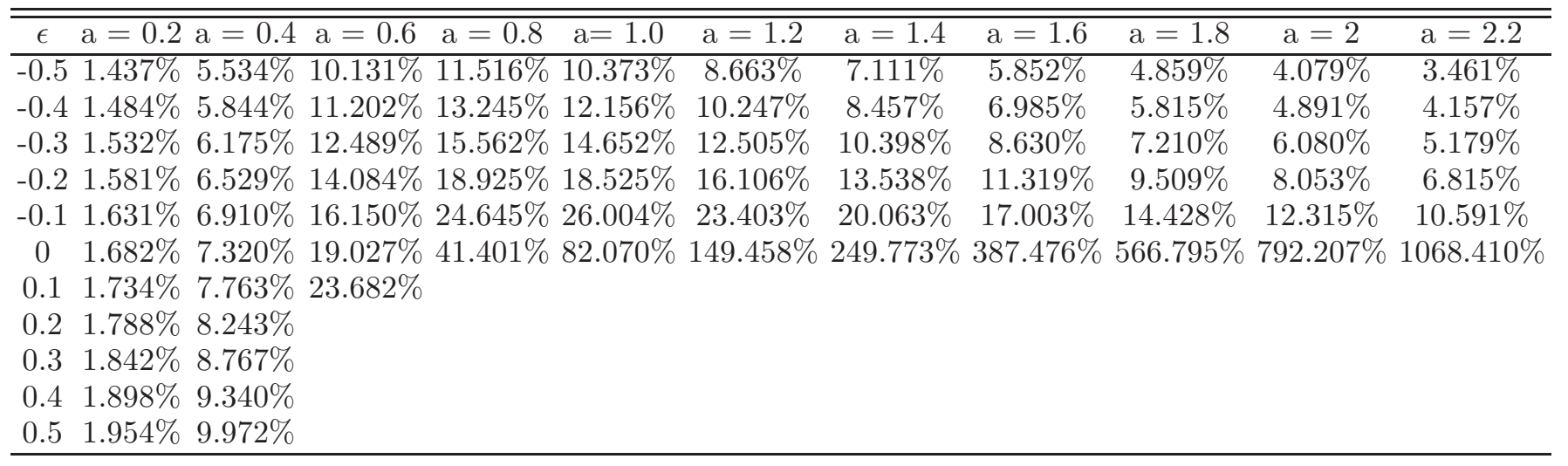


TABLE VI: The maximum energy efficiency $\eta_{\max }$ of energy extraction in the HD non-Kerr black hole for different values of deformation parameter $\epsilon$ corresponding to dimension $D=7$.

\begin{tabular}{cccccccccccc}
\hline \hline$\epsilon$ & $\mathrm{a}=0.25$ & $\mathrm{a}=0.5$ & 0.75 & $\mathrm{a}=1.0$ & $\mathrm{a}=1.25$ & $\mathrm{a}=1.5$ & $\mathrm{a}=1.75$ & $\mathrm{a}=2.0$ & $\mathrm{a}=2.25$ & $\mathrm{a}=2.5$ & $\mathrm{a}=2.75$ \\
\hline-0.5 & $2.362 \%$ & $8.112 \%$ & $11.476 \%$ & $10.581 \%$ & $8.573 \%$ & $6.779 \%$ & $5.392 \%$ & $4.348 \%$ & $3.562 \%$ & $2.960 \%$ & $2.494 \%$ \\
-0.4 & $2.448 \%$ & $8.738 \%$ & $13.062 \%$ & $12.415 \%$ & $10.204 \%$ & $8.133 \%$ & $6.501 \%$ & $5.261 \%$ & $4.320 \%$ & $3.597 \%$ & $3.035 \%$ \\
-0.3 & $2.537 \%$ & $9.438 \%$ & $15.111 \%$ & $14.970 \%$ & $12.544 \%$ & $10.106 \%$ & $8.134 \%$ & $6.614 \%$ & $5.450 \%$ & $4.550 \%$ & $3.847 \%$ \\
-0.2 & $2.628 \%$ & $10.227 \%$ & $17.922 \%$ & $18.891 \%$ & $16.298 \%$ & $13.344 \%$ & $10.852 \%$ & $8.889 \%$ & $7.363 \%$ & $6.173 \%$ & $5.236 \%$ \\
-0.1 & $2.722 \%$ & $11.127 \%$ & $22.193 \%$ & $26.237 \%$ & $23.925 \%$ & $20.185 \%$ & $16.736 \%$ & $13.900 \%$ & $11.635 \%$ & $9.834 \%$ & $8.394 \%$ \\
0 & $2.819 \%$ & $12.166 \%$ & $30.463 \%$ & $60.445 \%$ & $103.491 \%$ & $159.506 \%$ & $227.914 \%$ & $308.220 \%$ & $400 \%$ & $503.320 \%$ & $617.808 \%$ \\
0.1 & $2.918 \%$ & $13.389 \%$ & & & & & & & & & \\
0.2 & $3.021 \%$ & $14.860 \%$ & & & & & & & & & \\
0.3 & $3.126 \%$ & $16.693 \%$ & & & & & & & & & \\
0.4 & $3.234 \%$ & $19.101 \%$ & & & & & & & & & \\
0.5 & $3.345 \%$ & $22.601 \%$ & & & & & & & & & \\
\hline
\end{tabular}

[13] S. Chen and J. Jing, Phys. Lett. B 711, 81 (2012).

[14] T. Johannsen and Psaltis, Phys. Rev. D 83, 124015 (2011).

[15] E. T. Newman and A. I. Janis, J. Math. Phys. (N. Y.) 6, 915 (1965).

[16] N. Yunes and L. C. Stein, Phys. Rev. D 83, 104002 (2011).

[17] A. A. Abdujabbarov, B. J. Ahmedov, S. R. Shaymatov, and N. B. Jurayeva, Phys. Rev. D 87, 064042 (2013).

[18] T. A. Apostolatos, G. Lukes-Gerakopoulos, and G. Contopoulos, Phys. Rev. Lett. 103, 111101 (2009).

[19] C. Bambi and L. Modesto, Phys. Lett. B 711, 10 (2012).

[20] C. Bambi, Astrophys. J. 761, 174 (2012).

[21] F. Caravelli and L. Modesto, Phys. Lett. B 711, 10 (2012).

[22] C. Bambi, Phys. Rev. D 87, 023007 (2013).

[23] C. Bambi, J. Cosmol. Astropart. Phys. 08 (2013) 055.

[24] S. Chen, and J. Jing, Phys. Rev. D 85, 124029 (2012).

[25] T. Johannsen, Adv. Astron. 2012, 1 (2012).

[26] T. Johannsen, Phys. Rev. D 87, 124010 (2013).
[27] T. Johannsen, Phys. Rev. D 87, 124017 (2013).

[28] T. Johannsen, J. Phys. Conf. Ser. 410, 012136 (2013).

[29] R. A. Konoplya and A. Zhidenko, Phys. Rev. D 87, 024044 (2013).

[30] C. Liu, S. Chen, and J. Jing, Astrophys. J. 751, 148 (2012).

[31] T. Johannsen and D. Psaltis, Astrophys. J. 726, 11 (2011).

[32] R. Emparan and H. S. Reall, Living Rev. Relativity 11, 6 (2008).

[33] F. R. Tangherlini, Nuovo Cimento 27, 636 (1963).

[34] R. C. Myers and M. J. Perry, Ann. Phys. (N. Y.) 172, 304 (1986).

[35] R. P. Kerr, Phys. Rev. Lett. 11, 237 (1963).

[36] T. Johannsen and D. Psaltis, Astrophys. J. 716, 187 (2010).

[37] S. G. Ghosh and U. Papnoi, Preprint (2013).

[38] A. N. Aliev, Phys. Rev. D 74, 024011 (2006). 